\title{
Role of argon laser as an adjunctive therapy for treatment of resistant infected corneal ulcers
}

This article was published in the following Dove Press journal:

Clinical Ophthalmology

23 May 2014

Number of times this article has been viewed

\author{
Mohammad M Khater \\ Adel A Selima \\ Mohammad S El-Shorbagy \\ Ophthalmology Department, \\ Tanta University, Tanta, Egypt
}

Purpose: To evaluate the role of argon laser as an adjunctive therapy in ten patients with resistant infected corneal ulcers with or without hypopyon.

Methods: The study included 20 patients, split into two groups of ten, with resistant infected corneal ulcers with or without hypopyon. One group was considered as the control group and treated with local and systemic specific antimicrobial drugs guided with culture and sensitivity tests. The other group started with the same specific therapy as the control group for 1 week with no obvious improvement and then was further treated with argon laser. The ten patients in the control group included five cases of fungal ulcers, three mixed (fungal and bacterial) ulcers, and two viral ulcers. The ten patients in the other group included three cases of fungal ulcers, three mixed (fungal and viral) ulcers, three viral ulcers, and one bacterial ulcer as proven with microbial culture and sensitivity tests. Eight cases of the control group and seven cases of the other group were associated with hypopyon. Before laser treatment, a drop of benoxinate hydrochloride $0.4 \%$ and a single drop of fluorescein sodium $0.25 \%$ were instilled. Argon laser irradiation of the affected cornea was performed using an argon $532 \mathrm{~nm}$ wavelength (Carl Zeiss LSL 532s AG; Carl Zeiss Meditec AG, Jena, Germany). A spot size of $500 \mu \mathrm{m}$, pulse duration of 0.2 seconds, and power of $900 \mathrm{~mW}$ were used. All cases were followed up for 3 months after healing was achieved.

Results: During the first 4 weeks after laser treatment, all patients showed complete healing of the epithelial defect and resolution of stromal infiltration with no adverse effects. In the control group, four cases needed an amniotic membrane graft due to thinning and the other six cases were healed in a duration that ranged from 3 to 7 weeks.

Conclusion: Argon laser phototherapy is useful as an adjunctive treatment for resistant infected corneal ulcers. More cases are needed to get more reliable results and to confirm our findings.

Keywords: adjunctive treatment, hypopyon, infected keratitis

\section{Introduction}

Resistant infected corneal ulcer is a sight-threatening infection that may end in great visual impairment, like other types of stromal inflammation. ${ }^{1}$ The patient in many instances does not seek medical advice immediately or after taking many inappropriate medications that modify the clinical picture and so diagnosis is commonly delayed and the condition worsens and the infection becomes advanced. ${ }^{2}$ Management of resistant infected corneal ulcers is a challenging situation and no standards have been defined. ${ }^{3}$ The available specific agents are limited in their efficacy because of poor penetration into the cornea and the resistance of some species to those agents. ${ }^{4}$ The present study reports on the usefulness of argon laser phototherapy as an adjunctive therapy for resistant infected corneal ulcers.

\section{Patients and methods}

Patients in this study attended the Cornea unit in the Ophthalmology Department at Tanta University Hospital in Egypt for medical consultation in the period from February 2013 to August 2013. 
They were suffering from different forms of resistant infected corneal ulcers. They were divided into two groups, each of which included ten cases of resistant infected corneal ulcers with or without hypopyon. One group was considered as the control group and treated with local and systemic specific antimicrobial drugs guided by culture and sensitivity tests. The other group started the specific treatment according to culture and sensitivity as in the control group for 1 week and when no obvious improvement was achieved, the ten cases in this group were treated with argon laser. The ten cases of the control group included five fungal ulcers, three mixed (fungal and bacterial) ulcers, and two viral ulcers. The ten cases of the other group included three fungal ulcers, three mixed (fungal and viral) ulcers, three viral ulcers, and one bacterial ulcer as proven with microbial culture and sensitivity tests. Eight cases of the control group and seven cases of the laser treatment group were associated with hypopyon. Informed consent was obtained from every participant in this study.

In the first visit, a careful ophthalmological examination was done and a detailed history was taken from each patient in order to detect the predisposing factors that may trigger such cases and so be corrected if they are correctable. Also, the history informed us of previous medications that were taken. Then we stopped all specific drugs taken by the patients for 1 day and continued only the nonspecific drugs like atropine sulphate and lubricants to allow the chance for microbial culture and sensitivity tests to be done. Ocular ultrasonography was done routinely in every case to assess the posterior segment in order to detect its involvement, which may alter the approach of the treatment.

Slit lamp examination of each case was done stressing the size of the epithelial defect in both horizontal and vertical meridians, depth of the ulcer, density of the surrounding edema and infiltration, anterior chamber reaction, and level of hypopyon if present. The size of corneal ulcers ranged from $2-8 \mathrm{~mm}$ in both horizontal and vertical diameters and the depth of the ulcer did not exceed the upper half of the corneal stroma.

The culture and sensitivity results showed multiple microorganisms. Fungi including both hyphae and yeast like Candida albicans were demonstrated and also Gram positive bacteria such as Staphylococci and Streptococci and Gram negative bacteria as Haemophilus influenzae and pseudomonas aeruginosa were found.

According to the culture and sensitivity results, the specific antimicrobial drug was prescribed for each case depending on the highest organism sensitivity (highest number of positive results in the culture and sensitivity report). The antifungal drugs prescribed included amphotericin B 0.15\% (Fungizone; Bristol-Myers Squibb, New York, NY, USA) prepared as fortified drops, fluconazole $0.2 \%$ (Diflucan; Pfizer, Inc., New York, NY, USA) taken directly in an eye dropper from the vial, natamycin 5\% (Natamet; Sun Pharmaceutical Industries Limited, Mumbai, India), and itraconazole 1\% (Itral; Jawa Pharmaceuticals, Lahore, Pakistan). The antibiotics included gatifloxacin 0.5\% (Zymer; Allergan, Inc., Irvine, CA, USA), moxifloxacin 0.5\% (Vigamox; Alcon Laboratories, Inc., Fort Worth, TX, USA), ofloxacin 0.3\% (Oflox; Allergan, Inc.) and tobramycin $0.3 \%$ (Tobrex; Alcon Laboratories, Inc.). The antiviral drug used in viral cases was acyclovir 3\% (Zovirax; GalaxoSmithKline plc, London, UK).

Daily follow-up for 1 week was done in all cases of both groups regarding the size of the ulcer in both horizontal and vertical meridians, depth of the ulcer, surrounding edema and infiltration, anterior chamber reaction, and level of hypopyon if present. When improvement was achieved, we continued the medical treatment only; the case was included in the control group.

When no noticeable improvement was achieved after 1 week, the ulcer was considered resistant to treatment and argon laser therapy was tried as an adjunctive treatment to the usual corneal ulcer treatment including both specific and nonspecific measures; the case was included in the laser treatment group.

Before laser treatment, the eye was medicated with benoxinate hydrochloride $0.4 \%$ as surface anesthesia and a single drop of fluorescein sodium $0.25 \%$ was instilled in order to stain the corneal epithelial defect to allow the chance for argon laser energy to be absorbed by the stained corneal tissue because the argon laser beam is not absorbed with the unstained corneal tissue as in case of argon laser pan-retinal photocoagulation where the argon laser beam traverses the unstained cornea without absorption. Argon laser irradiation was performed using argon green wavelength (Carl Zeiss LSL 532s AG; Carl Zeiss Meditec AG, Jena, Germany). Argon laser beam parameters were spot size of $500 \mu \mathrm{m}$, pulse duration of 0.2 seconds, and power of $900 \mathrm{~mW}$. The argon laser beam was focused on the stained corneal epithelial defect and when triggered we noticed blanching of the corneal stroma and small cavitations that may have reached the middle stroma. The total area of epithelial defect was covered with argon laser shots in one session. These laser shots were triggered towards the corneal epithelial defect side by side to cover the whole area. The number of shots was dependent on the size of each ulcer; we covered the stained epithelial defect of the ulcer during treatment with laser shots and the 
number of laser shots ranged from 22-148 shots as shown on the device counter (missed shots due to eye movement in certain patients were included).

Follow-up of each case was done daily in order to detect the response to the argon laser therapy as adjunctive therapy as usual in corneal ulcer treatment until complete healing was achieved. In each case we assessed size of the epithelial defect, density and edge of infiltration, edema, depth of the ulcer, and level of hypopyon. Then, each case was followed up for 3 months to detect any relapse or recurrence.

\section{Statistics}

Statistical presentation and analysis of the present study was conducted using chi-square test with SPSS V.16 (SPSS Inc., Chicago, IL, USA).

\section{Results}

Clinical observation of the cases treated with argon laser revealed the following results (Figures 1-3):

1. Healing was achieved after about 2 weeks in six cases.

2. Healing was achieved after about 3 weeks in one case.

3. Healing was achieved after about 4 weeks in two cases.

4. One case did not improve after laser therapy and so after 1 week of follow-up, an amniotic membrane graft was done to help healing, which was repeated after another 1 week until complete healing was achieved after a total duration of 6 weeks from the first visit.

5. Reactivation happened in one viral ulcer (healing was achieved after about 2 weeks), recollection of hypopyon occurred after about 3 weeks from complete healing and an amniotic membrane graft was done to help healing, which was achieved after about 2 weeks from grafting.
From the above results, we found that:

1. $80 \%$ of cases healed without relapse.

2. $10 \%$ of cases healed with relapse.

3. $60 \%$ of cases healed after about 2 weeks from laser therapy.

4. $10 \%$ of cases healed after about 3 weeks from laser therapy.

5. $20 \%$ of cases healed after about 4 weeks from laser therapy.

6. Amniotic membrane grafting was needed in $20 \%$ of cases (total).

As regards the visual acuity results, we found that:

1. At the time of first presentation, nine cases ranged from hand motion to 0.2 (decimal system) and only one case achieved 0.7 at the first visit (peripheral viral ulcer).

2. No visual acuity improvement was achieved in four cases $(40 \%)$.

3. Visual acuity improvement was achieved with a gain of one line in three cases (30\%).

4. Visual acuity improvement was achieved with a gain of two lines or more in three cases (30\%).

The control group treated with specific antimicrobial drugs only, without argon laser therapy, showed the following results:

1. Healing was achieved after about 3 weeks in two cases $(20 \%)$.

2. Healing was achieved after about 4 weeks in one case $(10 \%)$.

3. Healing was achieved after about 6 weeks in two cases $(20 \%)$.

4. Healing was achieved after about 7 weeks in one case $(10 \%)$.
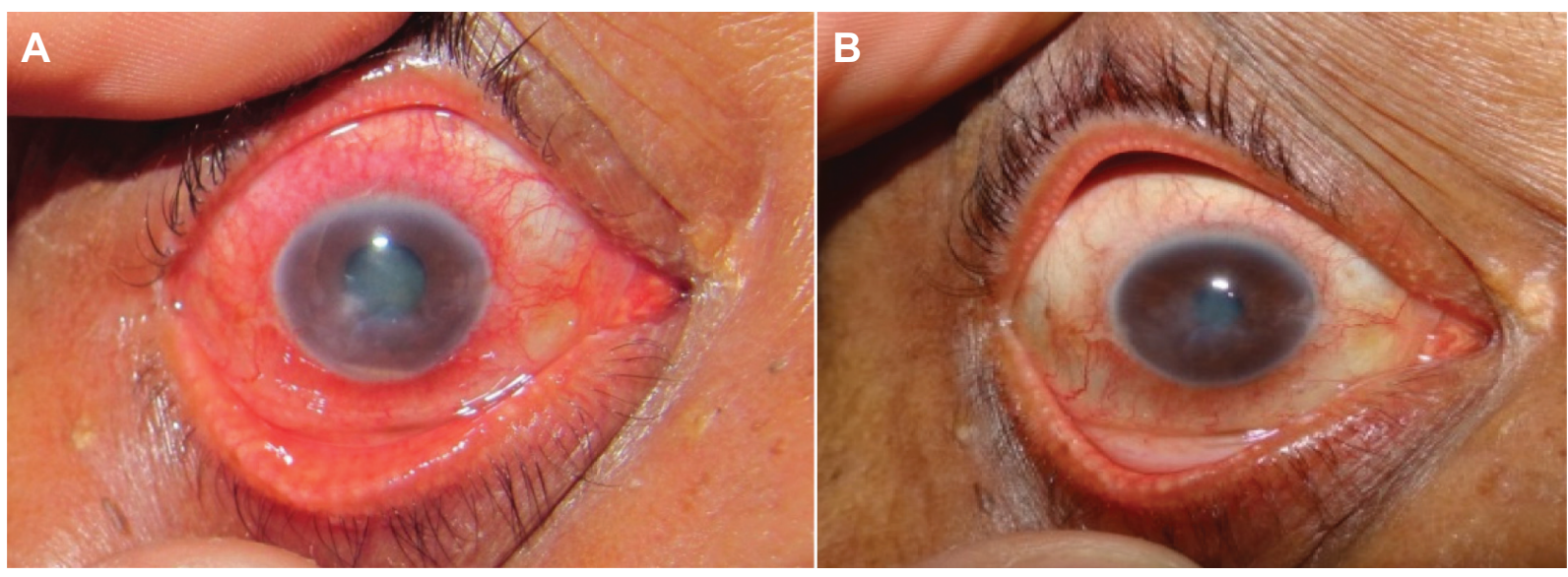

Figure I Resistant viral corneal ulcer associated with hypopyon before (A) and after (B) argon laser adjunctive therapy. 

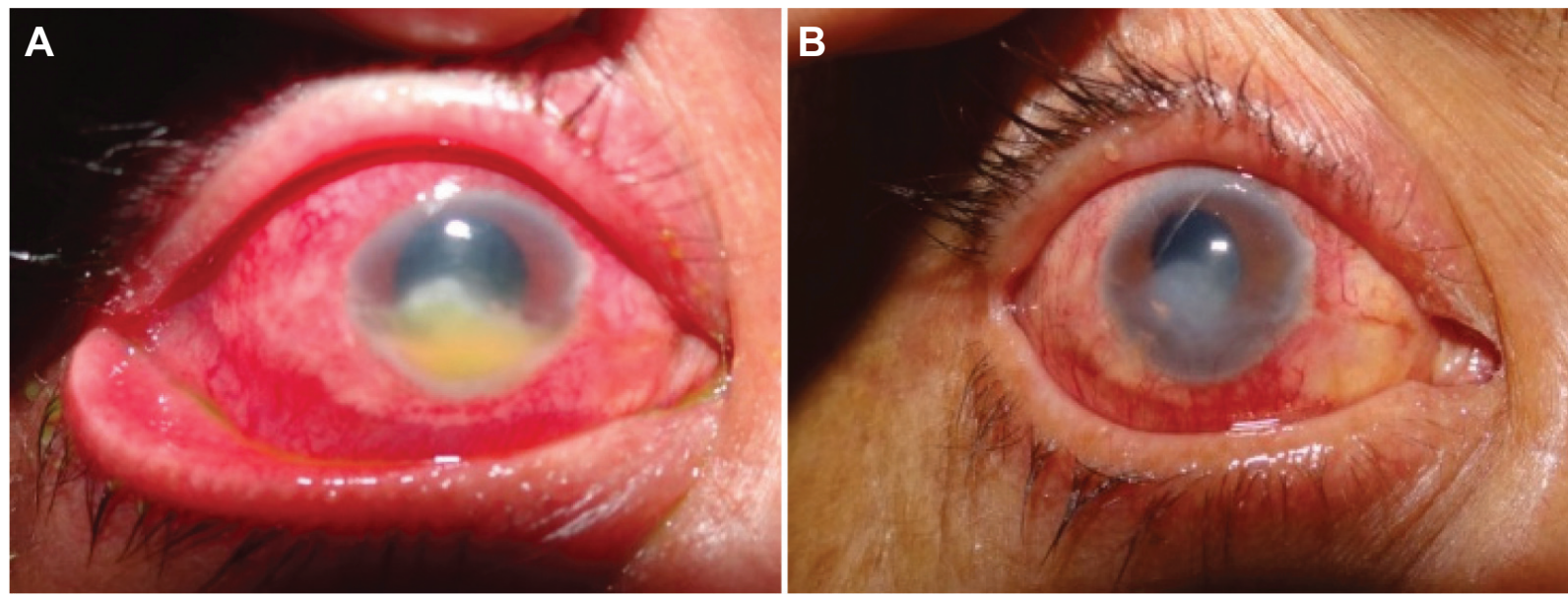

Figure 2 Resistant fungal corneal ulcer associated with hypopyon before (A) and after (B) argon laser adjunctive therapy.

5. Amniotic membrane grafting was needed in four cases $(40 \%)$.

As regards the visual acuity results in the control group, we found that:

1. At the time of first presentation, all cases ranged from hand motion to 0.1 .

2. No visual acuity improvement was achieved in seven cases $(70 \%)$.

3. Visual acuity improvement was achieved with a gain of one line in three cases (30\%).

When we compared the results of both groups we found that:

- As regards healing, nine cases healed without grafting in 2-4 weeks in the laser group, while only six cases healed without grafting in 3-7 weeks in the control group, with a significant $P$-value of 0.006 when comparing the two groups (Table 1, Figure 4).

- As regards visual acuity improvement, in the laser treatment group, three cases showed improvement with a gain of two or more lines, three cases with a gain of one line, and four cases showed no improvement; in the control group, three cases showed a gain of one line and seven cases showed no improvement; with a significant $P$-value when comparing the two groups (Table 2, Figure 5).

\section{Discussion}

Management of resistant corneal ulcers represents one of the great problems that face ophthalmologists all over the world. Many studies reported that surgical interference was the way adopted to find a solution for this problem. In the United States, $34 \%$ of patients required penetrating keratoplasty. In another series, penetrating keratoplasty was performed in $47 \%$ of the cases. ${ }^{5,6}$

Many specific drugs were used and prescribed for the treatment of resistant corneal ulcers according to the results of culture and sensitivity tests. Resistance to these drugs was reported in many cases when used alone and so adjunctive
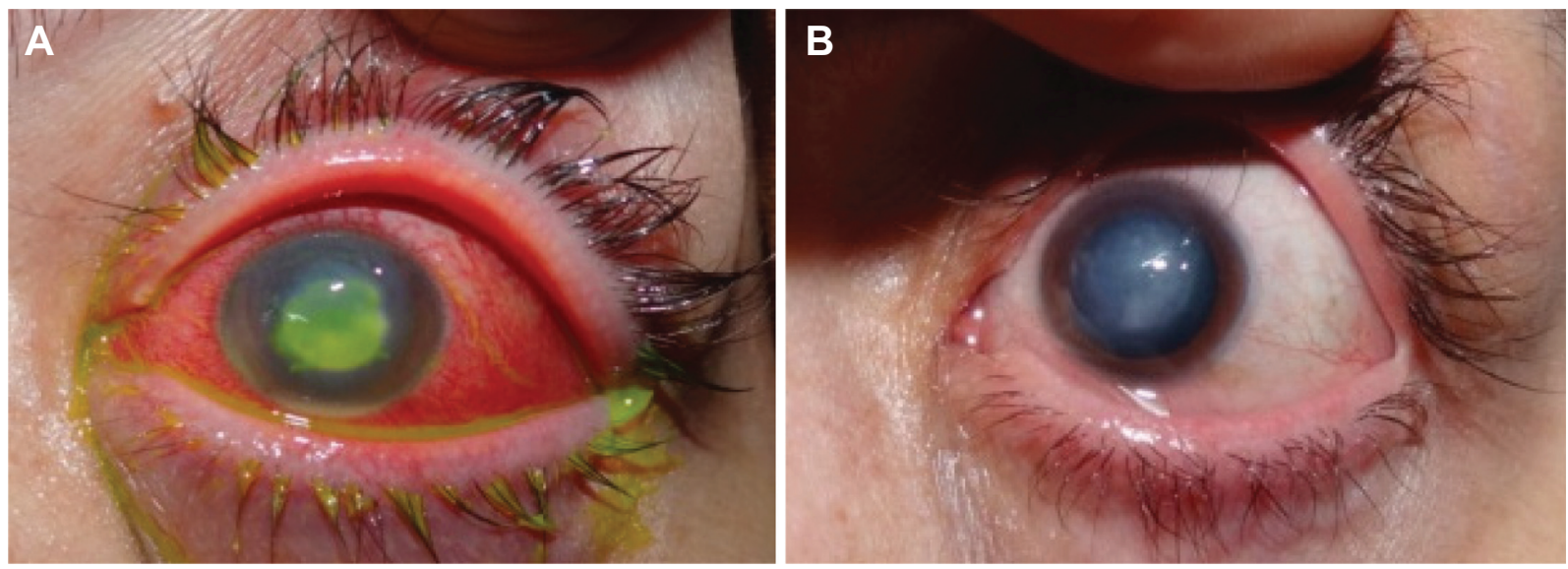

Figure 3 Resistant corneal ulcer associated with hypopyon related to contact lens before (A) and after (B) argon laser adjunctive therapy. 
Table I Percentage and duration of healing of resistant corneal ulcers in both laser and control groups

\begin{tabular}{lllll}
\hline Duration & Laser & Control & $\chi^{2}$ & P-value \\
\hline 2 weeks & $6(60 \%)$ & $0(0 \%)$ & 4.152 & $0.006^{*}$ \\
3 weeks & $1(10 \%)$ & $2(20 \%)$ & & \\
4 weeks & $2(20 \%)$ & $1(10 \%)$ & & \\
5 weeks & $0(0 \%)$ & $0(0 \%)$ & & \\
6 weeks & $0(0 \%)$ & $2(20 \%)$ & & \\
7 weeks & $0(0 \%)$ & $1(10 \%)$ & & \\
\hline
\end{tabular}

Note: *Significant result.

treatments were described. These adjunctive treatments included: subconjunctival injections of antifungal drugs like fluconazole and amphotericin B, intra-lesional stromal injection of voriconazole, crosslinking with exposure to ultraviolet radiation, amniotic membrane grafting, and conjunctival flaps. ${ }^{7-11}$

Lasers produce a coherent, focused, monochromatic, highenergy form of light. The wavelength of light emitted by the laser and the time sequence for delivery are important determining factors that obviously control the effect of laser light on eye tissue. Argon laser produces thermal damage of the cell through heating, denaturation, and coagulation of its protiens..$^{12,13}$

Epithelial debridement is frequently used in the management of fungal keratitis to enhance the penetration of antifungals due to the large area of keratitis. ${ }^{14}$ Argon laser may have the same effect. In addition, argon laser may have a fungicidal action because of the thermal effect on the infected tissue. It has been reported that the temperature in corneal tissue rises over 90 degrees after argon laser. ${ }^{15}$

To the best of our knowledge, there are no reports or retrograde studies that have investigated the treatment of resistant corneal ulcers with argon laser in humans. Fromer and L'Esperance reported the treatment of Pseudomonas keratitis in rabbit corneas by using argon laser in $1971 .^{16}$

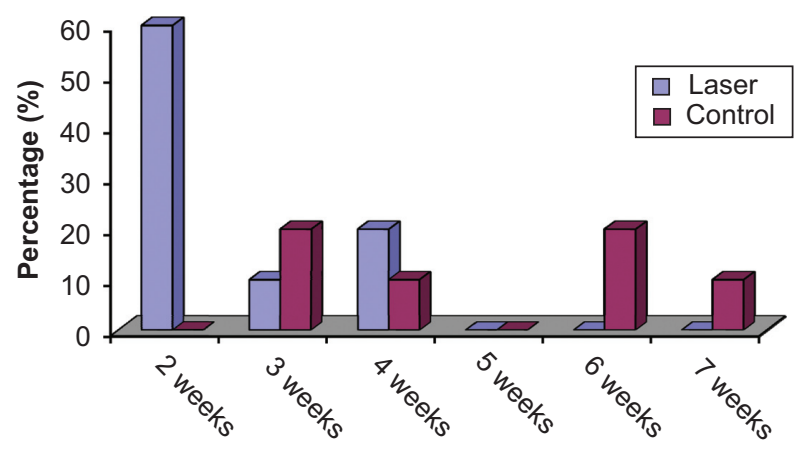

Figure 4 Percentage and duration of healing of resistant corneal ulcers in both laser and control groups.
Table 2 Visual acuity improvement in both groups

\begin{tabular}{lllll}
\hline & Laser & Control & $\chi^{2}$ & $P$-value \\
\hline No improvement & $4(40 \%)$ & $7(70 \%)$ & 3.82 & $0.014 *$ \\
Gain of one line gain & $3(30 \%)$ & $3(30 \%)$ & & \\
Gain of two or more lines & $3(30 \%)$ & $0(0 \%)$ & & \\
\hline Note: *Significant result. & & & &
\end{tabular}

In our study, the parameters of the argon laser used were adjusted to a power of $900 \mathrm{~mW}$, spot size of $500 \mu \mathrm{m}$, and a pulse duration of 0.2 seconds. Two signs were observed during the procedure: blanching of the cornea stroma and small cavitations that may have reached the middle stroma.

When the laser therapy procedure was combined with the specific antimicrobial agents according to the culture and sensitivity test results, the duration of the treatment was obviously shortened and this can be considered a good result. Complete healing was achieved in $80 \%$ of cases in the laser treatment group in a period ranging from $2-4$ weeks without the need for other adjunctive procedures. Only one case $(10 \%)$ needed amniotic membrane grafting to achieve complete healing. In the control group, healing without amniotic membrane graft was achieved in only six cases in a longer duration ranging from 3-7 weeks with a significant $P$-value of 0.006 when the results of both groups were compared using a chi-square test.

As regards the visual acuity results, improvement with one or more line gain (decimal system) was achieved in $60 \%$ of cases and four cases (40\%) did not show visual acuity improvement in the laser treatment group, while only three cases in the control group showed improvement with one line gain only. Also, a significant $P$-value of 0.014 was obtained when the visual acuity results were compared using a chi-square test.

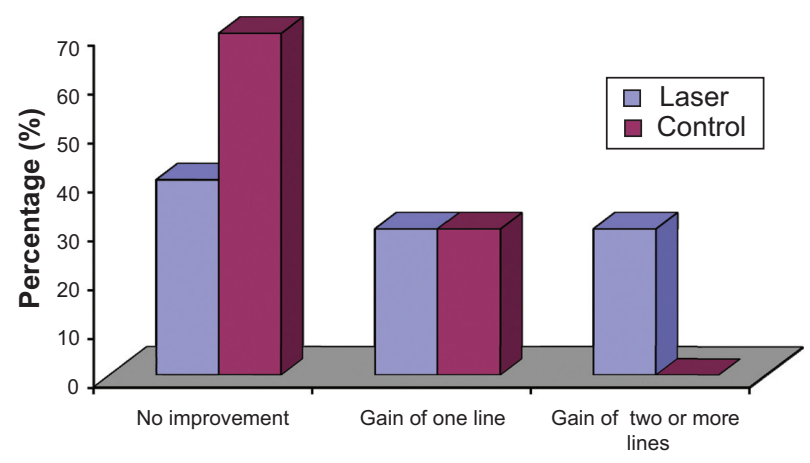

Figure $\mathbf{5}$ Visual acuity improvement in the laser and control groups after healing. 


\section{Disclosure}

The authors report no conflicts of interest in this work.

\section{References}

1. Tuli SS. Fungal keratitis. Clin Ophthalmol. 2011;5:275-279.

2. Thomas PA. Fungal infections of the cornea. Eye (Lond). 2003;17(8): 852-862.

3. Rautaraya B, Sharma S, Kar S, Das S, Sahu SK. Diagnosis and treatment outcome of mycotic keratitis at a tertiary eye care center in eastern India. BMC Ophthalmol. 2011;11:39.

4. Ou JI, Acharya NR. Epidemiology and treatment of fungal corneal ulcers. Int Ophthalmol Clin. 2007;47(3):7-16.

5. Patel A, Hammersmith K. Contact lens-related microbial keratitis: recent outbreaks. Curr Opin Ophthalmol. 2008;19(4):302-306.

6. Pradhan L, Sharma S, Nalamada S, Sahu SK, Das S, Garg P. Natamycin in the treatment of keratomycosis: correlation of treatment outcome and in vitro susceptibility of fungal isolates. Indian J Ophthalmol. 2011; 59(6):512-514.

7. Dev S, Rajaraman R, Raghavan A. Severe fungal keratitis treated with subconjunctival fluconazole. Am J Ophthalmol. 2006;141(4):783; author reply $783-784$.
8. Sharma N, Agarwal P, Sinha R, Titiyal JS, Velpandian T, Vajpayee RB. Evaluation of intrastromal voriconazole injection in recalcitrant deep fungal keratitis: case series. Br J Ophthalmol. 2011;95(12):1735-1737.

9. Carrasco MA, Genesoni G. Treatment of severe fungal keratitis with subconjunctival amphotericin B. Cornea. 2011;30(5):608-611.

10. Galperin G, Berra M, Tau J, Boscaro G, Zarate J, Berra A. Treatment of fungal keratitis from fusarium infection by corneal cross-linking. Cornea. 2012;31(2):176-180.

11. Yildiz EH, Nurozler AB, Ozkan Aksoy N, Altiparmak UE, Onat M, Karaguzel H. Amniotic membrane transplantation: indications and results. Eur J Ophthalmol. 2008;18(5):685-690.

12. Sandinha T, Zaher SS, Roberts F, Devlin HC, Dhillon B, Ramesh K. Superior fornicial conjunctival advancement pedicles (SFCAP) in the management of acute and impending corneal perforations. Eye (Lond). 2006;20(1):84-89.

13. Krauss JM, Puliafito CA, Steinert RF. Laser interactions with the cornea. Surv Ophthalmol. 1986;31(7):37-53.

14. Pellegrino F, Carrasco MA. Argon laser phototherapy in the treatment of refractory fungal keratitis. Cornea. 2013;32(1):95-97.

15. Loh AR, Hong K, Lee S, Mannis M, Acharya NR. Practice patterns in the management of fungal corneal ulcers. Cornea. 2009;28(8):856-859.

16. Fromer C, L'Esperance F. Argon laser phototherapy of pseudomonas corneal ulcers. Invest Ophthalmol. 1971;10(1):1-8.
Clinical Ophthalmology

\section{Publish your work in this journal}

Clinical Ophthalmology is an international, peer-reviewed journal covering all subspecialties within ophthalmology. Key topics include: Optometry; Visual science; Pharmacology and drug therapy in eye diseases; Basic Sciences; Primary and Secondary eye care; Patient Safety and Quality of Care Improvements. This journal is indexed on

\section{Dovepress}

PubMed Central and CAS, and is the official journal of The Society of Clinical Ophthalmology (SCO). The manuscript management system is completely online and includes a very quick and fair peer-review system, which is all easy to use. Visit http://www.dovepress.com/ testimonials.php to read real quotes from published authors. 